\title{
Application Experience of Moist Wound Healing Theory Combined with Modern New Dressings in the Treatment of Pressure Ulcers
}

\author{
Na Zhao*, Mingjuan Zhao, Yulan Zhao \\ Shandong Tai'an Municipal Hospital, Tai'an 271000, Shandong Province, China
}

*Corresponding author: Na Zhao, 624446433@qq.com

\begin{abstract}
Objective: Research on the effect of moist wound healing theory in a combination with modern new dressing treatment in patients diagnosed with pressure ulcers. Method: Selected 30 patients with pressure ulcers from our hospital, which is Shandong Tai'an Municipal Hospital, from January 2019 to January 2021 were divided into experimental group (15 cases, treated with moist wound healing theory combined with modern new dressings) and control group (15 cases, applied conventional treatments). The treatment effect, time of wound edema subsidence, wound healing time, number of dressing changes, granulation tissue growth time, and diameter reduction time were compared between the two groups. Results: The total effective rate of the experimental group $(93.33 \%, 14 / 15)$ was higher than that of the control group $(53.33 \%, 8 / 15)$, $\mathrm{P}<0.05$; the time to subsidence of wound edema in the experimental group was (3.11 \pm 0.22$)$, and the time for wound healing was (12.78 \pm 0.45$)$, the number of dressing changes (7.13 \pm 0.34$)$ times, the growth time of granulation tissue (5.43 \pm 2.22$)$, the diameter reduction time (6.25 \pm 3.75$)$, compared with the control group, $\mathrm{P}<0.05$. Conclusion: In the clinical treatment of patients diagnosed with pressure ulcers, the effective combination of moist healing theory and modern new dressing therapy has significant effects, whereby it speeds up the healing process of the pressure ulcers, and it is proven to be worthy to be promoted for usage.
\end{abstract}

Keywords: Moist wound healing theory; Modern new dressing; Pressure ulcers; Application

Publication date: July 2021; Online publication: July 31, 2021

\section{Introduction}

Pressure ulcers, also called pressure injuries, are mainly caused by local tissues being compressed for a long time, causing blood circulation in the compressed area to develop continuous ischemia and hypoxia. This means that it is unable to supply the skin and subcutaneous tissues with the required nutrients, resulting in the loss of local tissues. The basic function is broken and becomes necrotic ${ }^{[1]}$. Pressure ulcers are generally common in patients who have been bedridden for a long time, such as spinal cord injury, coma, and elderly femoral neck fractures. Pressure ulcers will increase the suffering of patients and make nursing work more difficult. If they cannot be cured for a long time, there is the possibility of complicated infections, and eventually death due to circulation failure. It can be seen that in-depth research and analysis of the clinical treatment plan for patients with pressure ulcers have a certain significance.

\section{Materials and methods}

\subsection{Baseline data}

Thirty admitted patients with pressure ulcers in our hospital from January 2019 to January 2021 were selected as the research objects. 15 patients were randomly selected to be included in the control group and 
received routine treatment. The remaining patients were classified as the experimental group and combined with moist wound healing theory modern new dressing treatment. The control group had 10 males, 5

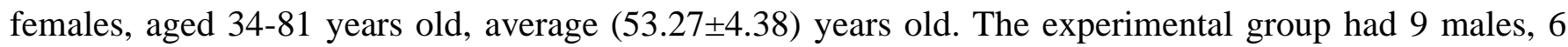
females, 36-79 years old, average (53.21) \pm 4.33 ) years old. The basic conditions of the two groups of patients were similar, with no statistical significance $(\mathrm{P}>0.05)$.

\subsection{Method}

Communicated with both the groups of patients, and developed a general nutritional support to remove local compression. Pressure ulcer wounds were cleaned with normal saline and the wounds were washed [2].

The control group was treated with conventional treatment. The patients used petroleum jelly gauze and gauze to wrap the wounds, and the dressing was changed every day. If the wound is covered with black scab, necessary protective measures should be taken for conservative observation.

The experimental group applied moist wound healing theory combined with modern new dressing treatments. Before changing the dressings, patients should be observed and their pressure ulcers evaluated. The assessment content mainly included pressure ulcer's time of observation, wound site, size, shape and stage, as well as margins, epithelial tissues, etc. Peripheral tissues, necrotic tissues, infections, pain, exudate volume, etc., were also observed.

\subsubsection{The treatment process is reflected in the following aspects}

First, the preparation of wound bed. Combined the color characteristics of the wound surface of pressure ulcers, classified and deal it with various types of pressure ulcers. Then, identified and cleared the wound healing barrier according to the overall requirements of wound preparation for the wound healing process to speed up. Among the 15 patients, three were in the black stage, four were in the yellow stage, seven were in the red stage, and one was in the powder stage. Combining different stages of pressure ulcer wound base difference to determine dressing and dressing plan ${ }^{[3]}$.

Second, the black wound period. The main symptom of the patient's wound base was necrosis and avascular tissue, the actual healing speed was slow, and bacterial proliferation was prone to occur. At that stage, the wound was mainly black, tan, yellow or brown, so in the clinical treatment, efforts were made to remove necrotic tissue and reduce the infection rate. Debridement was carried out according to the doctor's advice, combined with surgical debridement and autolytic debridement. Then applied hydrogel debridement on the crust, and changed the medicine once a day or every other day. During dressing change, removed the loosened scabs until they fall off. Subsequently, dressings were selected according to the new stage of the wound and a dressing change plan was made. Among them, Kanghuier debridement glue was an active hydrophilic material with a water content of $96 \%$. It can form a semi-solid substance, which was similar to gel and it was soft. It uses sodium carboxymethyl cellulose as the main component. It was used after contact with the wound to ensure moderate humidity on the wound surface, which was conducive to wound healing ${ }^{[4]}$. In addition, Kanghuier debridement gel can form a closed and slightly acid moist environment, which was conducive to the proliferation of fibroblasts and blood vessels.

During the yellow wound period, the base of the wound was yellow rotten flesh or necrotic tissue, which allowed bacteria to obtain growth conditions. Therefore, during treatment, carrion was removed and infection was prevented, the growth rate of granulation was accelerated, and the exudate was absorbed. After cleaning the wound, scraped out and cut off the yellow rotten flesh. When necessary, applied local anesthesia before removing it. The moist wound dressings were silver ion dressing, beauty salt dressing, and foam dressing from the inside to the outside. The dressing was changed every day or every other day. If the wound has been infected or was suspected of being infected, the frequency of dressing changes was appropriately increased, and airtight dressings were not selected. Among them, the metal silver layer of the 
silver ion dressing will release anions after contact with the exudate to kill bacteria. The effect can last about 7 days, but the silver ion dressing should not be used for a long time, and the time can be controlled within 2-4 weeks ${ }^{[5]}$. The salt dressing contains sodium oxide hypertonic salt, which can provide a hypertonic environment for the wound, absorb the exudate from the wound, and at the same time control granulation edema and speed up healing. The foam dressing has a strong ability to absorb exudate and has low permeability. The wound can always be kept in a moist state without adhesion to the wound. The effect of using it in superficial wounds was ideal.

Fourth, during the red wound period, the wound bed of the pressure ulcer covered the granulation tissue and was pale pink. When the granulation tissue thickens, the color changed to red, similar to beef. At that time, it was necessary to protect the new granulation tissue, prepare for epithelialization, and at the same time absorb a large amount of exudate, thereby the wound surface was always in a moist state. In addition, an effective moist dressing was selected according to wound exudation and granulation growth. In general, alginate dressings and foam dressings were suitable for low to moderate exudation. The reason was that there was calcium alginate in the alginate dressing, which absorbs the exudate in a short time and keep the wound surface at the best healing humidity. Calcium and sodium ions may be replaced into gels, which then adsorbed and processed necrotic tissues and bacteria, speeding up wound hemostasis. If the amount of exudate was small and the wound was relatively dry, hydrogel dressings and hydrocolloid dressings were used as the support, and the medicine was changed 1-2 times a week ${ }^{[6]}$.

Fifth, the pink wound period refers to the final healing period of pressure sores. At that stage, the foundation was almost filled with granulation tissue, and the epithelium was in a state of proliferation and crawling, which appeared pink. The focus of the treatment of pink wounds was to comprehensively protect the newly appeared epithelial tissue, which was more conducive to epithelialization. Generally, the dressing can be of hydrocolloid, foam or sheet hydrogel type, and the medicine needed to be changed every week, the number of times was 1-2 times. Among them, the sheet-shaped hydrogel dressing contains $60 \%$ of physiological saline, which was conducive to obtaining necessary water from the wound surface, providing necessary assistance for epithelial crawling, and being able to absorb a small amount of exudate.

\subsection{Evaluation Index}

(1) Evaluate the treatment effect of the two groups.

(2) Comparative analysis of patients' wound edema subsidence time, wound healing time, number of dressing changes, granulation tissue growth time, and diameter reduction time.

\subsection{Statistical analysis}

Data processing, SPSS23.0 statistical software. Data description, counting data is ( $\mathrm{n} \%)$, measurement data is $(\overline{\mathrm{X}} \pm \mathrm{s})$. Difference test, counting data is $\mathrm{X}^{2}$, measurement data is t. Statistical significance criterion, $\mathrm{P}<0.05$.

\section{Results}

3.1. Analysis of the treatment effect of the experimental group and the control group Index comparison between groups, $\mathrm{P}<0.05$. (Table 1.).

\subsection{Comparison of treatment indicators between the two groups}

Compared with the control group, the data of the experimental group was $\mathrm{P}<0.05$. (Table 2.). 
Table 1. Study on the treatment effect of two groups of patients (n/\%)

\begin{tabular}{ccccccc}
\hline Group & n & cure & Excellent & Valid & Invalid & Effective rate \\
\hline Test group & 15 & 9 & 4 & 1 & 1 & 93.33 \\
Control group & 15 & 5 & 2 & 1 & 7 & 53.33 \\
$\mathrm{X}^{2}$ & & & & & & 6.1364 \\
$\mathrm{P}$ & & & & & & 0.0132 \\
\hline
\end{tabular}

Table 2. Comparison of the treatment indicators of the experimental group and the control group ( $\overline{\mathrm{x}} \pm \mathrm{s}$ )

\begin{tabular}{ccccccc}
\hline Group & $\mathbf{n}$ & $\begin{array}{c}\text { Wound } \\
\text { edema } \\
\text { subsidence } \\
\text { time }\end{array}$ & $\begin{array}{c}\text { Wound } \\
\text { healing } \\
\text { time }\end{array}$ & $\begin{array}{c}\text { Switching } \\
\text { frequency }\end{array}$ & $\begin{array}{c}\text { Granulation } \\
\text { tissue } \\
\text { growth time }\end{array}$ & $\begin{array}{c}\text { Diameter } \\
\text { reduction } \\
\text { time }\end{array}$ \\
\hline Test group & 15 & $3.11 \pm 0.22$ & $12.78 \pm 0.45$ & $7.13 \pm 0.34$ & $5.43 \pm 2.22$ & $6.25 \pm 3.75$ \\
Control group & 15 & $5.72 \pm 0.34$ & $19.79 \pm 0.58$ & $13.34 \pm 0.52$ & $10.28 \pm 3.86$ & $14.37 \pm 4.62$ \\
T value & & 24.9611 & 36.9836 & 38.7118 & 4.2184 & 5.2852 \\
P value & & 0.0000 & 0.0000 & 0.0000 & 0.0002 & 0.0000 \\
\hline
\end{tabular}

\section{Discussion}

Pressure ulcer patients have a long illness with many complications and slow recovery. Patients with pressure ulcers based on a variety of diseases will have other diseases which threatens the lives of the patients if they do not take reasonable treatments. For this reason, in the clinical treatment of patients with pressure ulcers, assessment work should be carried out according to specific nursing procedures, and nursing plans should be formulated and revised, as well as relevant treatment plans should be actively implemented ${ }^{[7]}$. Among them, the choice of new dressings should be combined with wound characteristics, contraindications and indications. Modern moist dressings are formed on the basis of moist wound healing theory, and it is believed that the optimal moist environment is more conducive to the growth of fibroblasts and epidermal cells, thereby accelerating wound healing. Compared with traditional dressings, moist dressings can provide a moist environment for the wound to prevent the dressing from adhering to the wound, which is conducive to the formation and epithelialization of granulation. It also reduces the propagation and spread of bacteria, shortens the healing time of the wound, and effectively reduces the chance of infection, thus relieving the patient's conscious pain ${ }^{[8]}$.

In this study, patients in the experimental group were treated with moist wound healing theory combined with modern new dressings, and the moist wound healing theory was integrated into the dressing process, and compared with the control group, $\mathrm{P}<0.05$. This proves that in the process of clinical treatment of pressure ulcer patients, choosing the corresponding modern moist dressing according to the degree of wound recovery of the patient is more conducive to the healing of pressure ulcer wounds, and the feasibility of promotion is significant.

In general, during the clinical treatment of patients with pressure ulcers, the use of modern moist 
dressings has a significant effect, which can effectively prevent the infection rate of patients, reduce local pressure, ensure that the microcirculation is in a smooth state, and have a positive protective effect on the skin. Therefore, this method is highly clinically promoted, as it has an excellent application value.

\section{Disclosure statement}

The author declares no conflict of interest.

\section{References}

[1] Ying H, Wei H, Li W, 2017, Application of Wet Healing Therapy in Severe Pressure Ulcers of Stage III IV. Modern Clinical Nursing, 16(8): 46-48.

[2] Li H, Li S, 2020, Comparison of the Repair Effects of Collagen Dressing and Moist Burn Ointment on Wound Skin After Minimally Invasive Surgery for Underarm Odor. Chinese Journal of Experimental Surgery, 37(5): 973.

[3] Zhang Z, Li Z, Li Y, et al., 2021, Application of Alginate-Based Hydrogel/Dressing in Wound Healing: Continuous, Dynamic and Sequential Release. Chinese Tissue Engineering Research, 25(4) :638-643.

[4] Guo L, Zhang Q, Wang L, et al., 2020, Evaluation of the Effect of Negative Pressure Sealing Drainage and Wet Healing Technique in the Treatment of Hypospadias in Children. Chinese Journal of Plastic Surgery, 36(2): 180-184.

[5] Huang R, Niu C, et al., 2020, Net Meta Analysis of the Efficacy of Five Commonly used Dressings in the Treatment of Pressure Ulcers. Chinese Tissue Engineering Research, 24(16): 2614-2619.

[6] Chen X, Wu C, Zhang W, et al., 2020, The Effect of Silver Ion Antibacterial Dressing Combined with Pressure Ulcer Nursing on IL-6 and IL-8 in Patients with Wet Wound Healing. Modern Medicine and Health Research (Electronic Edition), 4 (1):170-171.

[7] Zeng Y, 2019, Comparison of the Efficacy of Compound Huangbai Liquid Combined with Microoxygen and Wet Dressings in the Treatment of Patients with Stage III and IV pressure ulcers. Guangdong Pharmaceutical University.

[8] Chen L, Liu M, Li L, 2020, Application of Local Vibration Therapy Combined with Pressure Ulcer Nursing in Patients with Pressure Injury. Zhejiang Clinical Medicine, 22(3):441-442,444. 